\title{
Approximation of ambiguity covariance matrix for integer de-correlation procedure in single-epoch GNSS positioning
}

\author{
Slawomir Cellmer \\ University of Warmia and Mazury in Olsztyn, Poland
}

\begin{abstract}
An ambiguity resolution plays the key role in GNSS carrier phase processing. Recently the LAMBDA method is widely used for this purpose. The algorithm of this method contains integer de-correlation procedure. An ambiguity covariance matrix is input data in this procedure. This matrix should to be positive definite. In traditional approach the observation data has to be complemented with additional group of code observations. It is necessary to obtain 'positive definiteness' in the case of single-epoch positioning. In the article new formula for approximation of positive definite ambiguity covariance matrix is presented. This formula does not require code observations. The numerical tests were carried out in order to show the efficiency of proposed solution. The results of these tests are presented.
\end{abstract}

Keywords: GNSS data processing; single-epoch positioning; ambiguity resolution.

\section{Introduction}

A single-epoch positioning is recently one of the biggest challenges in the research area concerning GNSS data processing. Many researchers have been developing this topic since last two decades, e.g., [1-9]. They have proposed different approaches for single-epoch positioning solution. Some approaches are based on regularization techniques used in solving ill-posed problem, e.g. [3], [10-11]. Other approaches apply widelane combinations in computational process [2], [4-6] proposed implementing widelane combinations in MAFA method to solve single-epoch positioning problem. However most of approaches apply LAMBDA method for ambiguity resolution [12]. This method includes de-correlation procedure. The full rank ambiguity covariance matrix (ACM) is required for this procedure. Unfortunately in the case of single-epoch positioning the ACM is rank deficient, when using only carrier phase data. In order to overcome this problem the simple formula of approximation of the ACM is derived and tested in this paper. The second section contains derivation of formula on computing the ACM matrix in the case of single-epoch positioning. This formula contains the $k$ parameter, which should be estimated. An algorithm of $k$-parameter estimation is presented in the third section. Next section contains an example of single-epoch solution using proposed approach. Then the results of other tests are presented and discussed. Finally some conclusions have been derived.

\section{An estimator of the modified ambiguity variance-covariance matrix}

In this paper is used a general formula of the ACM, which was derived in [5]. The observation equation of DD carrier phase is:

$$
\mathbf{L}+\mathbf{V}=\mathbf{A} \mathbf{X}+\mathbf{B N}
$$

where:

$\mathbf{L}$ - vector of DD carrier phase observations in cycles

$\mathbf{V}$ - residuals vector in cycles

A - design matrix for real valued parameters (baseline components)

$\mathbf{X}$ - vector of real valued parameters [m]

B - design matrix for ambiguities

$\mathbf{N}$ - ambiguity vector in cycles.

Corresponding author: Slawomir Cellmer. E-mail address: slawomir.cellmer@gmail.com

http://dx.doi.org/10.3846/enviro.2014.198

(C) 2014 The Author. Published by VGTU Press. This is an open-access article distributed under the terms of the Creative Commons Attribution License, which permits unrestricted use, distribution, and reproduction in any medium, provided the original author and source are credited. 
Let us assume for simplicity that the A matrix is $n \times 3$, where $n$ is a number of observations (only coordinates are assumed as parameters). The LS solution of the equations (1) is obtained from the following normal equations:

$$
\left[\begin{array}{ll}
\mathbf{A}^{\mathrm{T}} \mathbf{P A} & \mathbf{A}^{\mathrm{T}} \mathbf{P B} \\
\mathbf{B}^{\mathrm{T}} \mathbf{P A} & \mathbf{B}^{\mathrm{T}} \mathbf{P B}
\end{array}\right]\left[\begin{array}{l}
\mathbf{X} \\
\mathbf{N}
\end{array}\right]=\left[\begin{array}{l}
\mathbf{A}^{\mathrm{T}} \mathbf{P L} \\
\mathbf{B}^{\mathrm{T}} \mathbf{P L}
\end{array}\right],
$$

where $\mathrm{P}$ is an observation weight matrix.

From (2) follows the general form of ACM [5]:

$$
\mathbf{Q}_{\mathrm{NN}}=\left[\mathbf{B}^{\mathrm{T}} \mathbf{P B}-\mathbf{B}^{\mathrm{T}} \mathbf{P A}\left(\mathbf{A}^{\mathrm{T}} \mathbf{P A}\right)^{-1} \mathbf{A}^{\mathrm{T}} \mathbf{P B}\right]^{-1},
$$

This matrix is applied in the LAMBDA method as a basis for the integer de-correlation procedure. In the case of a single epoch positioning, $\mathbf{B}$ is an identity matrix $(\mathbf{B}=\mathbf{I})$ and the ACM (3) should be obtained as an inverse of the following matrix:

$$
\mathbf{P}_{\mathrm{NNa}}=\mathbf{P}-\mathbf{P A}\left(\mathbf{A}^{\mathrm{T}} \mathbf{P A}\right)^{-1} \mathbf{A}^{\mathrm{T}} \mathbf{P} .
$$

Unfortunately, this matrix is not positive definite. Therefore, it does not satisfy the condition required for the LAMBDA method. Nevertheless, matrix (4) can be replaced by its approximation, which is positive definite. Cellmer in [5] proposed to assume additional group of observation in the model (1). These observations have nature of code observations but another weight matrix. They are assumed to exist only for the purpose to form appropriate approximation of ACM. In such a case the approximation of ACM can be written as:

$$
\mathbf{Q}_{\mathrm{NNb}}=\left[\mathbf{B}_{0}^{\mathrm{T}} \mathbf{P}_{0} \mathbf{B}_{0}-\mathbf{B}_{0}^{\mathrm{T}} \mathbf{P}_{0} \mathbf{A}_{\mathbf{0}}\left(\mathbf{A}_{0}^{\mathrm{T}} \mathbf{P}_{0} \mathbf{A}_{0}\right)^{-1} \mathbf{A}_{0}^{\mathrm{T}} \mathbf{P}_{0} \mathbf{B}_{0}\right]^{-1},
$$

where $\mathbf{B}_{\mathbf{0}}, \mathbf{A}_{\mathbf{0}}$ and $\mathbf{P}_{\mathbf{0}}$ are the following block matrices:

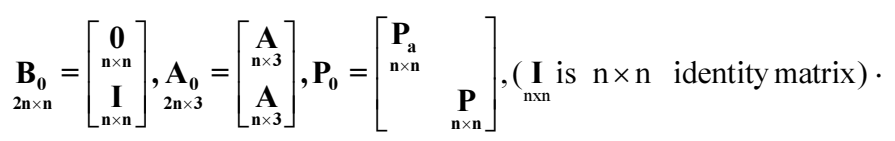

$\mathbf{P}_{\mathbf{a}}$ is a weight matrix of the simulated, additional observations. It is assumed here that design matrix $\mathbf{A}$ for simulated observations is the same as for real observations, and $\mathbf{B}$ is a null matrix for these observations. The weights of simulated observations must be small enough so that their impact on the results is negligible. On the other hand, they must assure the positive definiteness of the ACM. If we assume that the structures of the matrices $\mathbf{P}_{\mathrm{a}}$ and $\mathbf{P}$ are the same, then:

$$
\mathbf{P}_{a}=c \mathbf{P},
$$

where $c$ is a positive coefficient less than one $(0<c<1)$.

Taking into account (6) and (7) the formula (5) can be rewritten as:

$$
\mathbf{Q}_{\mathrm{NNb}}=\left[\mathbf{P}-k \mathbf{P A}\left(\mathbf{A}^{\mathrm{T}} \mathbf{P A}\right)^{-1} \mathbf{A}^{\mathrm{T}} \mathbf{P}\right]^{-1}, \text { where }: \quad k=\frac{1}{1+c} .
$$

The $\mathbf{Q}_{\mathbf{N N b}}$ matrix is then used as a base for de-correlation procedure [12]:

$$
\mathbf{Q}_{\mathrm{Nz}}=\mathbf{Z} \mathbf{Q}_{\mathrm{NNb}} \mathbf{Z}^{\mathbf{T}}
$$

where:

$\mathbf{Z}$ - integer de-correlation matrix

$\mathbf{Q}_{\mathbf{N}}-$ ambiguity covariance matrix

$\mathbf{Q}_{\mathbf{N z}}-$ diagonal transformed ambiguity covariance matrix.

In summary, the simulation of additional observations is equivalent to the use of the coefficient $k$ as in (8). The formula (8) presents the estimator of modified ACM in the case of single epoch positioning.

\section{An algorithm of optimal $k$-coefficient estimation}

It should be noticed, that the model (6) is in fact the same as if code observations were used along with the phase observations. The one significant difference is a way of the weighting of the simulated observations. The nominal standard deviation of code observations is about one hundred times lower than the nominal standard deviation of phase observations. Thus, in the standard model, the weights for code observations are 10000 times smaller than the weights of the phase observations. The $k$ coefficient corresponding to such (standard) solution would be equal to 0.9999. Cellmer in [5] has proposed to fix the coefficient $\mathrm{c}=0.01$ (the weights for simulated observations are 100 times smaller than weights for real data). Hence, the coefficient $k$ equals 0.99 . It was empirically tested by author that value $k=0.99$ gives better results than standard value $\mathrm{k}=0.9999$. It should be also mentioned that the $k$ coefficient can be derived empirically based on the test of 
stochastic properties of GNSS observations performed by the way of the zero baseline test [13] or the laboratory tests [14]. However the optimal value of $k$ can vary depend on satellite configuration (or other factors included in a functional model represented by the A matrix). Therefore it is proposed in this paper to apply the value of k coefficient that depends on the model. The proposed method of $\mathrm{k}$ coefficient estimation is based on analysis of ACM eigenvalues. ACM has to be positive defined. Usually this property is tested by checking eigenvalues. They should be positive. However, in case of weakly conditioned models (as in single-epoch positioning) the positive eigenvalues can be complex numbers. Therefore, it is proposed here to examine an additional property of ACM: whether all eigenvalues are real. Thus the estimation of $k$ coefficient is carried out according to the following procedure:



Fig. 1. Flowchart of the $k$-coefficient estimation

We start with standard value of $k=0.9999$. Then ACM is computed from (8). After that, eigenvalues of $\mathrm{Q}_{\mathrm{NNb}}$ are computed. Next step is testing whether all eigenvalues are real. If one or more eigenvalues are complex numbers then $k$ coefficient is reduced by $0.0001: k=k-0.0001$ and all steps are repeated starting from computing $\mathrm{Q}_{\mathrm{NNb}}$ matrix. If all eigenvalues are real, then the $\mathbf{Q}_{\mathbf{N N b}}$ matrix is used as ACM for LAMBDA method.

Thus, summarizing this section, the $k$-coefficient estimation is carried out according to flowchart presented on Figure 1. Final value of $k$-coefficient is used to compute ACM from (8). This matrix is applied to the de-correlation procedure in the Lambda method.

\section{The example}

The computational process of single epoch positioning was carried out. An approximate position was obtained from singleepoch positioning DGPS. This position was in the distance of $2.276 \mathrm{~m}$ away from actual position. Next, the double differenced carrier phase observations were used as a data. Due to a poor a priori position, the widelane combination (L1-L2) were applied. Two different values of $k$ coefficient were considered. The final results reveal significant impact of $\mathrm{k}$ value on solution. Even in the cases as the values of $k$ coefficient vary only 0.0001 . Table 1 contains the comparison of results for $k=0.9999$ and for $k=0.9998$. In the first row the values of $k$ coefficient are listed. The second row contains $\mathrm{Q}$ matrices computed basing on (8). In the third row eigenvalues are listed. The fourth row contains transformation matrices obtained from integer de-correlation procedure (9). The fifth row contains residuals of ambiguities computed as differences between values obtained from single-epoch solution and the actual values of ambiguities. The last row contains the distance between positions of single-epoch solution and actual solution computed as:

$$
\mathrm{V}_{\mathrm{d}}=\sqrt{\mathrm{dX^{2 } + d \mathrm { Y } ^ { 2 } + \mathrm { dZ }}}
$$

where: $\mathrm{dX}, \mathrm{dY}, \mathrm{dZ}$ are differences between actual and computed coordinates.

As we can see in the case of $k=0.9999$ two eigenvalues are complex numbers. Their imaginary parts are very small (6.06e-17). In the case of $k=0.9998$ all eigenvalues are real numbers. The transformation matrices $\mathrm{Z}$ are different for these two cases. The final results are correct only in the case of $k=0.9998$. Thus reducing the $k$ coefficient by the value of 0.0001 has allowed to obtain correct solution. 
Table 1. The comparison of results for different values of $k$ factor

\begin{tabular}{|c|c|c|}
\hline$k$ & 0.9999 & 0.9998 \\
\hline \multirow[t]{6}{*}{ Q } & $\begin{array}{llllll}6.6215 & 4.3420 & 2.5202 & 4.8362 & 4.5312 & 4.6058\end{array}$ & $\begin{array}{llllll}3.3108 & 2.1710 & 1.2602 & 2.4181 & 2.2656 & 2.3029\end{array}$ \\
\hline & $\begin{array}{llllll}4.3420 & 6.9564 & 4.6032 & 4.9858 & 2.7613 & 4.3131\end{array}$ & $\begin{array}{llllll}2.1710 & 3.4783 & 2.3016 & 2.4928 & 1.3807 & 2.1566\end{array}$ \\
\hline & $\begin{array}{llllll}2.5202 & 4.6032 & 3.1601 & 3.4651 & 1.7614 & 2.2519\end{array}$ & $\begin{array}{llllll}1.2602 & 2.3016 & 1.5803 & 1.7326 & 0.8808 & 1.1260\end{array}$ \\
\hline & $\begin{array}{llllll}4.8362 & 4.9858 & 3.4651 & 5.6014 & 3.9565 & 2.2410\end{array}$ & $\begin{array}{llllll}2.4181 & 2.4928 & 1.7326 & 2.8008 & 1.9783 & 1.1206\end{array}$ \\
\hline & $\begin{array}{llllll}4.5312 & 2.7613 & 1.7614 & 3.9565 & 3.5438 & 2.0972\end{array}$ & $\begin{array}{llllll}2.2656 & 1.3807 & 0.8808 & 1.9783 & 1.7721 & 1.0487\end{array}$ \\
\hline & $\begin{array}{llllll}4.6058 & 4.3131 & 2.2519 & 2.2410 & 2.0972 & 5.8755\end{array}$ & $\begin{array}{llllll}2.3029 & 2.1566 & 1.1260 & 1.1206 & 1.0487 & 2.9379\end{array}$ \\
\hline \multirow[t]{6}{*}{$\mathrm{D}$} & 11.8787 & 5.9395 \\
\hline & 2.0000 & 1.0000 \\
\hline & 2.0000 & 1.0000 \\
\hline & 0.0002 & 0.0002 \\
\hline & $0.0002+6.06 \mathrm{e}-17 \mathrm{i}$ & 0.0002 \\
\hline & $0.0002-6.06 \mathrm{e}-17 \mathrm{i}$ & 0.0002 \\
\hline \multirow[t]{6}{*}{$Z$} & $\begin{array}{llllll}-7 & 4 & 3 & 0 & -2 & 0\end{array}$ & $\begin{array}{llll}-7 & 4 & -2 & -3\end{array}$ \\
\hline & $\begin{array}{llllll}3 & 3 & -4 & 4 & -3 & 1\end{array}$ & $\begin{array}{llllll}-1 & 3 & -3 & 4 & 4 & 1\end{array}$ \\
\hline & $\begin{array}{llllll}-7 & -5 & 4 & -5 & -1 & -2\end{array}$ & $\begin{array}{llllll}-2 & -5 & -1 & -4 & -5 & -2\end{array}$ \\
\hline & $\begin{array}{llllll}3 & 2 & 2 & 0 & 6 & 1\end{array}$ & $\begin{array}{llllll}3 & 2 & 6 & -2 & 0 & 1\end{array}$ \\
\hline & $\begin{array}{cccccc}5 & -6 & -5 & 0 & -3 & -1\end{array}$ & $\begin{array}{llllll}5 & -6 & -3 & 5 & 0 & -1\end{array}$ \\
\hline & $\begin{array}{llllll}3 & -2 & 0 & -1 & 3 & 0\end{array}$ & $\begin{array}{llllll}4 & -2 & 3 & 0 & -1 & 0\end{array}$ \\
\hline$\Delta \mathrm{a}$ & 4533325 & $\begin{array}{lllllllllll}0 & 0 & 0 & 0 & 0 & 0\end{array}$ \\
\hline $\mathrm{V}_{\mathrm{d}}$ & $9.488 \mathrm{~m}$ & $0.090 \mathrm{~m}$ \\
\hline
\end{tabular}

\section{Test results}

The proposed approach was tested using the real GPS data of three baselines. The data come from campaign performed in order to monitor local deformation in open-pit mine „Adamów” in Central Poland. This project is managed by Dr. Radoslaw Baryla from the Department of Satellite Geodesy and Navigation of University of Warmia and Mazury in Olsztyn. Figure 2 depicts the location of the measurement area and the layout of baselines. One GPS station of ASG-EUPOS, Polish part of European Positioning System active geodetic network [15-16], was used in test surveys ("KONI"). The surveys were performed on December 9th, 2008, on $30.7 \mathrm{~km}, 3.2 \mathrm{~km}$ and $0.5 \mathrm{~km}$ baselines, with a 30-second sampling rate. Data sets of each baseline consisted of 120 epochs. The data were processed independently for each epoch. The approximate position was computed using code-observations in DGPS mode basing on single reference station [17-18]. Next the double differences of carrier phase observations were formed. A data set was composed of L1-L2 widelane combinations. The ambiguity covariance matrix was formed according to formula (8), as a basis for the de-correlation procedure. The "true" coordinates were derived using Bernese software based on an 8-hour data set [19-20].
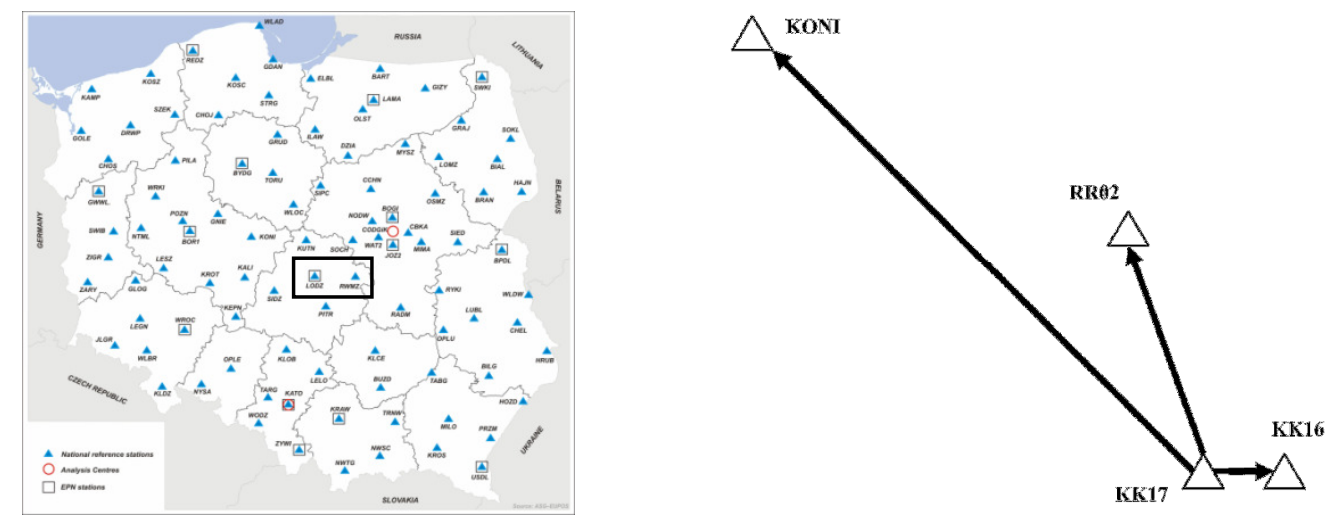

Fig. 2. The location of the test surveys http://www.asgeupos.pl/webpg/graph/dwnld/map_pl_EN.jpg

Figure 3 presents the comparison of two scenarios of 120 independent single-epoch solutions: for $k=$ const $=0.9999$, and alternatively for $k$-coefficient estimated according to proposed method. The bars on Figure 3 depict the linear residuals of the position obtained independently in each epoch, with respect to the "true" position. These residuals 
were computed by (10). The red lines relate to the solutions with correct ambiguities, the black lines to solutions with incorrect ambiguities. The blue lines depict the linear residuals of a priori position in each epoch obtained from DGPS. In most cases, a priori position was distant over $1 \mathrm{~m}$ from the actual position. For each case the percentage of correct solutions is shown in a text box. In the case of $0.5 \mathrm{~km}$ baseline, there was obtained $88(73 \%)$ correct solutions, among all 120 solutions for constant $\mathrm{k}$ coefficient, and 90 (75\%) correct solutions if $k$ coefficient was estimated according to proposed approach. In the case of $3.2 \mathrm{~km}$ baseline the results were adequately: $83(69 \%)$ for $k=0.9999$ and 86 (72\%) for $k$ estimated. In the case of the longest baseline $(30.7 \mathrm{~km})$, the results were: $73(61 \%)$ for $k=0.9999$ and $79(66 \%)$ for $k$ estimated. In each case the improvement was obtained on the level of a few percent ( $2-5 \%$ depending on baseline length).

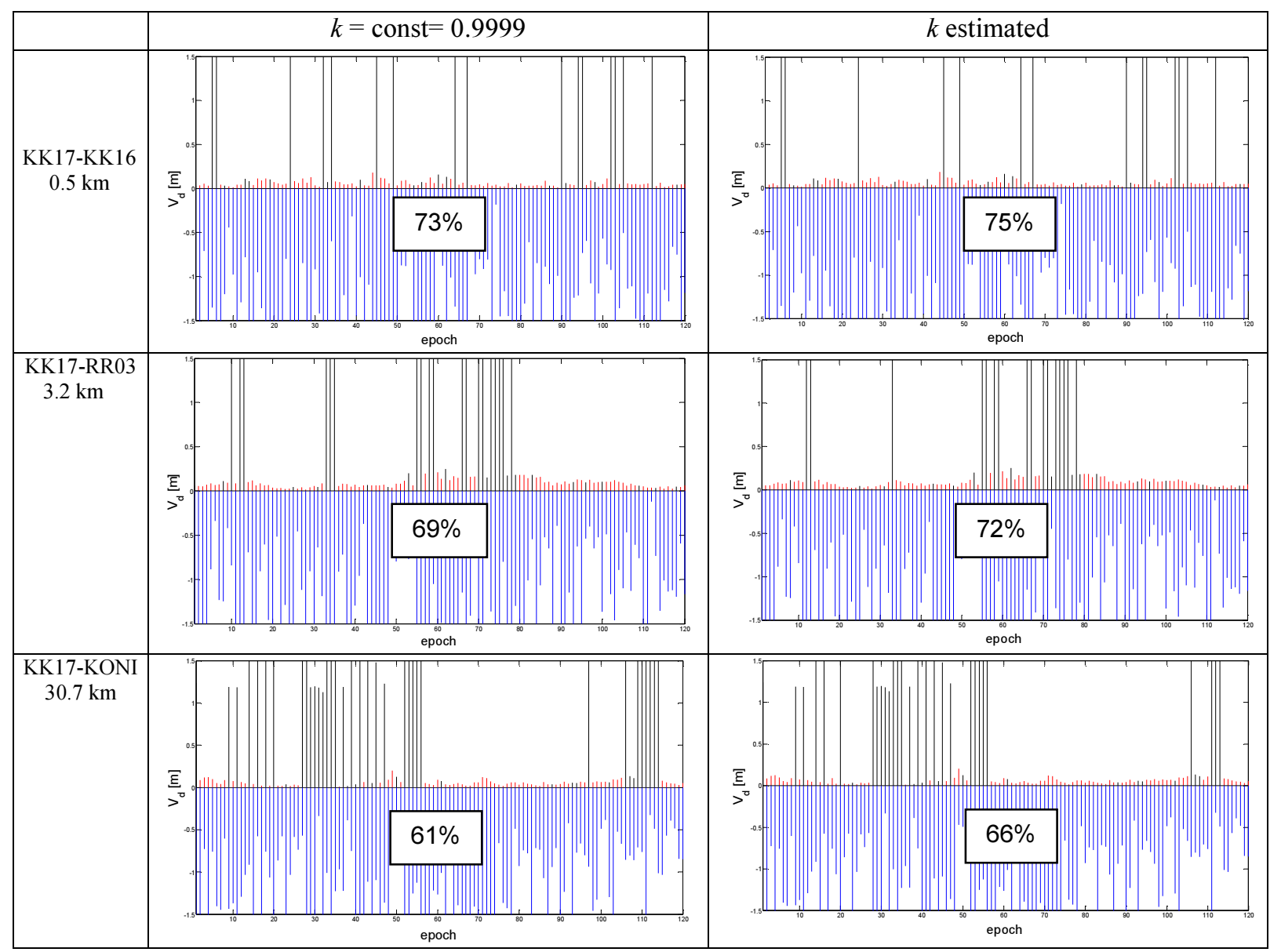

Fig. 3. The results of the tests of single-epoch positioning, for different scenarios of determining $k$ coefficient

\section{Conclusions}

A mathematical model of precise single-epoch positioning was discussed in the paper. It was shown that computational process in this model can be improved using a new method of approximation of ambiguity covariance matrix. This matrix is a base for integer de-correlation procedure, which is a part of Lambda method of ambiguity resolution. The approach presented in the paper is an alternative to traditional approach in which both code- and carrier phase observations are applied to derive covariance matrix in the case of single-epoch positioning. The tests have shown that there is possible to obtain good results by proposed approach, when in the same time traditional approach fails. Thus, this approach can be useful tool for increasing the success rate of single-epoch positioning. The improvement of efficiency was confirmed by the tests: in each case the success rate has increased when proposed approach has been applied. The aim of the tests was not to derive quantitative measures describing proposed approach. They were carried out to reveal potential possibilities of improving computational process in the case of single-epoch positioning. The results of the tests indicate that such possibilities exist definitely.

\section{References}

[1] Corbett, S. J.; Cross, P. A. 1995. GPS single epoch ambiguity resolution, Survey Review 33(257): 149-160. http://dx.doi.org/10.1179/sre.1995.33.257.149

[2] Lin, S.; Tzeng, D. 2006. Single Epoch Kinematic GPS Positioning Technique in Short Baseline, Journal of Surveying Engineering $132(2)$ : 52-57. http://dx.doi.org/10.1061/(ASCE)0733-9453(2006)132:2(52) 
[3] Wang, Z.; Rizos, C.; Lim, S. 2006. Single epoch algorithm based on tikhonov regularization for deformation monitoring using single frequency GPS Receivers, Survey Review 38(302): 682-688. http://dx.doi.org/10.1179/sre.2006.38.302.682

[4] Cellmer, S. 2011. The real time precise positioning using MAFA method, in The $8^{\text {th }}$ International Conference ENVIRONMENTAL ENGINEERING, selected papers, Vol. III, Vilnius, 1310-1314.

[5] Cellmer, S. 2012. On-the-fly ambiguity resolution using an estimator of the modified ambiguity covariance matrix for the GNSS positioning model based on phase data, Artificial Satellites 47(3): 81-90. http://dx.doi.org/10.2478/v10018-012-0015-9

[6] Cellmer, S. 2013. Search procedure for improving Modified Ambiguity Function Approach, Survey Review 45(332): 380-385. http://dx.doi.org/10.1179/1752270613Y.0000000045

[7] Paziewski, J.; Wielgosz, P. 2013. Assessment of GPS + Galileo and multi-frequency Galileo single-epoch precise positioning with network corrections, GPS Solutions. http://dx.doi.org/10.1007/s10291-013-0355-3

[8] Zinas, N.; Parkins, A.; Ziebart, M. 2013. Improved network-based single-epoch ambiguity resolution using centralized GNSS network processing, GPS Solutions 17: 17-27. http://dx.doi.org/10.1007/s10291-012-0256-x

[9] Paziewski, J.; Wielgosz, P.; Krukowska, M. 2013. Application of SBAS pseudorange and carries phase signals to precise instantaneous singlefrequency positioning, Acta Geodynamica et Geomaterialia 10(4): 421-430. http://dx.doi.org/10.13168/AGG.2013.0041

[10] Liu, G.; Zhu, Y.; Zhou, R. 2005. A new approach of single epoch GPS positioning for landslide monitoring, Acta seismologica sinica 18(4): 427-434. http://dx.doi.org/10.1007/s11589-005-0020-1

[11] Li, B.; Shen, Y.; Feng, Y. 2010. Fast GNSS ambiguity resolution as an ill-posed problem, Journal of Geodesy 84: 683-698. http://dx.doi.org/10.1007/s00190-010-0403-5

[12] Teunissen, P. 1995. The least-squares ambiguity decorrelation adjustment:a method for fast GPS integer ambiguity estimation, Journal of Geodesy 70: 65-82. http://dx.doi.org/10.1007/BF00863419

[13] Amiri-Simkooei, A. R.; Tiberius, C. C. J. M. 2007. Assessing receiver noise using GPS short baseline time series, GPS Solutions 11: 21-35. http://dx.doi.org/10.1007/s10291-006-0026-8

[14] Szpunar, R.; Dróżdż, M.; Próchniewicz, D. 2012. Analysis of the pseudoranges determined by means of GSG-54 generator, Electrical Review $88(9 \mathrm{a} / 2012): 230-234$

[15] Dawidowicz, K. 2013. Impact of different GNSS antenna calibration models on height determination in the ASGEUPOS network: a case study, Survey Review 45(332): 386-394. http://dx.doi.org/10.1179/1752270613Y.0000000043

[16] Bosy, J. 2013. Global, Regional and National Geodetic Reference Frames for Geodesy and Geodynamics, Pure and Applied Geophysics. Basel, Switzerland ( in print, published on-line): 1-26 URL: http://link.springer.com/article/10.1007/s00024-013-0676-8\# (available on January 2014) http://dx.doi.org/10.1007/s00024-013-0676-8

[17] Dawidowicz, K.; Krzan, G. 2014. Accuracy of single receiver static GNSS measurements under conditions of limited satellite availability, Survey Review.

[18] Bakuła, M. 2010. Network code DGPS positioning and reliable estimation of position accuracy, Survey Review 42(315): 82-91. http://dx.doi.org/10.1179/003962610X12572516251448

[19] Wielgosz, P.; Paziewski, J.; Baryla, R. 2011. On constraining zenith tropospheric delays in processing of local GPS networks with Bernese software, Survey Review 43(323): 472-483. http://dx.doi.org/10.1179/003962611X13117748891877

[20] Stępniak, K.; Baryła, R.; Wielgosz, P.; Kurpiński, G. 2013. Optimal data processing strategy in precise GPS leveling networks, Acta Geodynamica et Geomaterialia 10(4/172): 443-452. 